\title{
Adenosine induced PR jump on surface ECG to differentiate atrioventricular nodal re-entrant tachycardia from concealed accessory pathway mediated tachycardia: a bedside test
}

\author{
S C Toal, B U Vaijfdar, A K Gupta, A M Vora, Y Y Lokhandwala
}

Heart 2002;87:37-40

See end of article for authors' affiliations

Correspondence to:

Dr YY Lokhandwala,

Department of Cardiology,

KEM Hospital, Parel,

Mumbai 400012, India;

yashlokhandwala@

hotmail.com

Accepted

8 October 2001

\begin{abstract}
Objective: To evaluate the efficacy of single dose intravenous adenosine in differentiating atrioventricular nodal re-entrant tachycardia (AVNRT) from concealed pathway mediated atrioventricular re-entrant tachycardia (AVRT) using surface ECG at the bedside.

Method: $12 \mathrm{mg}$ of adenosine was administered to 97 consecutive patients who had documented narrow QRS tachycardia without manifest pre-excitation. The test was labelled positive for AVNRT if surface ECG recordings showed signs of dual atrioventricular (AV) node physiology-namely, PR jump or AV nodal echo. The diagnostic value of this test was evaluated by electrophysiological study as the yardstick.

Results: The adenosine test was positive for AVNRT in 48 patients (adenosine induced PR jump in 48, AV nodal echo in 3) and negative in 49 patients. On electrophysiological study, 62 patients had AVNRT and 35 had concealed pathway mediated AVRT. Thus, the test had a sensitivity of $74 \%$ and specificity of $94 \%$. The positive predictive value was $96 \%$ and the negative predictive value was $67 \%$. Conclusion: Single dose (12 mg) intravenous adenosine administered during sinus rhythm can identify dual AV node physiology on surface ECG recording at the bedside. A positive adenosine test identified by a PR jump can differentiate AVNRT from AVRT with a high specificity and positive predictive accuracy.
\end{abstract}

A denosine is an endogenous nucleoside whose effects on the heart by intravenous administration were identified in 1929 by Drury and Szent-Gyorgyi. ${ }^{1}$ Interest in adenosine, however, was kindled in 1955 when Somlo ${ }^{2}$ reported the successful treatment of paroxysmal supraventricular tachycardia with adenosine $5^{\prime}$ triphosphate (ATP). Adenosine is increasingly being considered to be the first line treatment to terminate paroxysmal supraventricular tachycardias. ${ }^{3}$ Its clinical use has been expanding not only from being a therapeutic option but also as a diagnostic tool to identify the mechanism in wide as well as narrow QRS tachycardias. ${ }^{45}$

Narrow QRS tachycardias comprise atrial tachycardias, atrioventricular nodal re-entrant tachycardias (AVNRT), atrioventricular re-entrant tachycardias (AVRT), and sinus node re-entrant tachycardias. Atrial tachycardias can usually be diagnosed from a 12 lead ECG. While few patients of AVRT have a manifest pre-excitation on ECG, the majority have concealed accessory pathways and hence no pre-excitation. It is often difficult to differentiate such concealed pathway mediated AVRT from AVNRT on the surface ECG.

Since AVNRT depends on dual atrioventricular (AV) nodal pathways, ${ }^{6}$ evidence of dual physiology, especially with AV nodal echo, practically makes the diagnosis of AVNRT very likely. ATP and adenosine have been used to diagnose dual AV node physiology in the electrophysiology laboratory. ${ }^{7}$ ATP in incremental doses has been used for the non-invasive diagnosis of concealed accessory pathways. ${ }^{8}$ However, a single dose bedside intravenous adenosine test for differentiating AVNRT from AVRT has not been described. Hence we planned to evaluate the efficacy of such a test, which can become a valuable diagnostic tool.

\section{METHODS}

\section{Study population}

Between October 1998 and November 1999, all consecutive patients referred to the Department of Cardiology, KEM
Hospital, Mumbai, India, for electrophysiological study (EPS) and radiofrequency ablation treatment for documented narrow QRS tachycardia without manifest pre-excitation were enrolled for this prospective study. Informed written consent was obtained from all patients before inclusion in the study. The study was granted approval by the ethics committee of the hospital. All antiarrhythmic drugs were withdrawn five half lives before the study.

Exclusion criteria were manifest pre-excitation on surface ECG, atrial arrhythmia, and contraindication to adenosine (bronchial asthma).

\section{The adenosine test}

A basal 12 lead ECG was recorded. Twelve milligrams of adenosine was injected into the antecubital vein as a rapid bolus. This was followed by a flush with $20 \mathrm{ml}$ of normal saline injected rapidly. ECG at a paper speed of $50 \mathrm{~mm} / \mathrm{s}$ was recorded continuously from the time of injection of adenosine until the heart rate returned to baseline. The surface ECG was monitored for changes in the PR interval, development of AV block, manifestation of pre-excitation, initiation of tachycardia, or the presence of retrograde $\mathrm{P}$ waves (AV nodal echo). Only a single dose of adenosine was given. Two independent cardiologists evaluated the ECG recording.

PR jump was defined as a sudden increase in PR interval by $\geqslant 50 \mathrm{~ms}$ between two consecutive sinus beats ( fig $\mathrm{l}$ ).

$A V$ nodal echo was defined as a retrograde $\mathrm{P}$ wave at the end of the QRS complex (fig 2).

The occurrence of a PR jump or AV nodal echo is a marker of dual AV node physiology. The test was labelled positive if

Abbreviations: ATP, adenosine $5^{\prime}$ triphosphate; AV, atrioventricular; AVNRT, atrioventricular nodal re-entrant tachycardia; AVRT, atrioventricular re-entrant tachycardia; EPS, electrophysiological study 


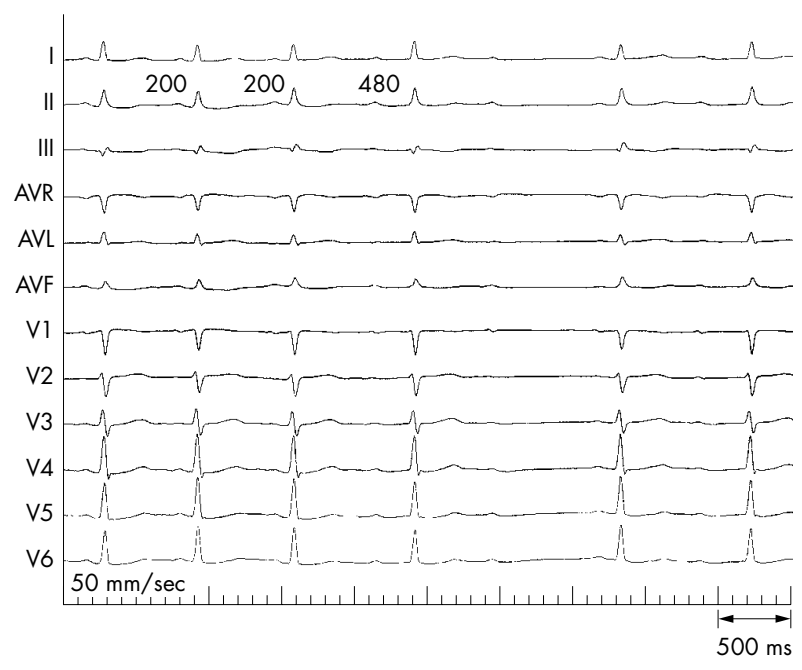

Figure 1 Twelve lead ECG after adenosine bolus injection showing a PR jump. PR intervals are measured in milliseconds. In the grid shown at the right lower corner, one small square $=100 \mathrm{~ms}$.

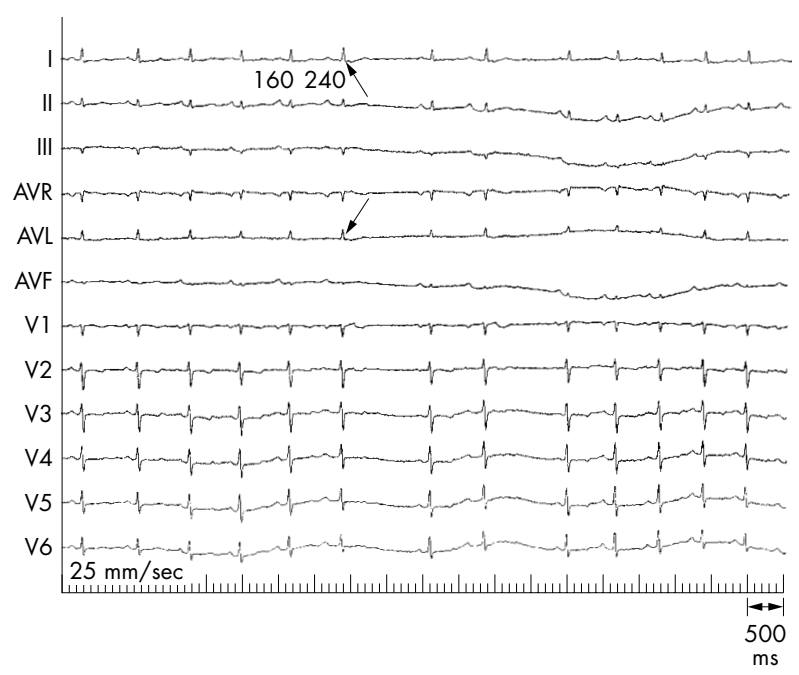

Figure 2 Atrioventricular nodal echo (retrograde $\mathrm{P}$ wave at the end of the QRS complex) during the adenosine test, shown by an arrow, which is preceded by a PR jump (denoted by numbers in milliseconds).

either of these were observed after injection of adenosine. If neither occurred, the test was labelled negative.

Patients with a positive test were presumed to have AVNRT while those with a negative test were considered to have AVRT.

\section{Electrophysiological study}

All patients underwent EPS using standard techniques and protocols. The results of EPS were considered to be the yardstick for establishing the mechanism of tachycardia as AVNRT or AVRT.

Dual AV node physiology was diagnosed if there was an AH jump, defined as an increment of $50 \mathrm{~ms}$ or more in the $\mathrm{AH}$ value during a $10+1$ atrial stimulation protocol with a basal cycle length of $500 \mathrm{~ms}$ or $430 \mathrm{~ms}$ and a decrement of $10 \mathrm{~ms}$ with each subsequent extrastimulus, pacing from high right atrium or coronary sinus. This protocol was repeated after radiofrequency ablation in all the patients to look for dual AV node physiology, which could have been masked by tachycardia before the ablation.

Absence of dual AV node physiology was defined as the failure to find an AH jump by atrial extrastimulation until "AV nodal refractoriness" was reached.
Table 1 Evidence of dual atrioventricular nodal physiology by adenosine challenge

\begin{tabular}{llll}
\hline Adenosine test result & AVNRT & AVRT & Total \\
\hline Positive & $46(74 \%)$ & $2(6 \%)$ & 48 \\
Negative & $16(26 \%)$ & $33(94 \%)$ & 49 \\
Total & 62 & 35 & 97
\end{tabular}

AVNRT, atrioventricular nodal re-entrant tachycardia; AVRT, atrioventricular re-entrant tachycardia

Tachycardia was induced in all patients to determine its mechanism.

\section{Statistical analysis}

The validity of the test was analysed by determining the sensitivity, specificity, and positive and negative predictive values of the test. Categorical variables were analysed by $\chi^{2}$ test and continuous variables were analysed by $t$ test. A probability value of $p<0.05$ was considered significant.

\section{RESULTS}

A total of 97 patients were included in the study. There were 40 men and 57 women. Their ages ranged from 12-62 years with a mean (SD) of 43 (12) years. All patients had a structurally normal heart except one who had a corrected transposition of the great arteries. There was 100\% concurrence in the findings of the two cardiologists who evaluated the ECG tracings.

PR jump was found in 48 patients, of whom three also had an AV nodal echo. No patient without a PR jump had an AV nodal echo. These 48 patients, with dual AV node physiology shown by the adenosine test, were labelled as having AVNRT and the remaining 49 as having AVRT. On EPS, using standard criteria, 62 patients were found to have AVNRT and the remaining 35 had AVRT.

With the use of the EPS as a yardstick to confirm the mechanism of tachycardia, the adenosine test was found to give a "true positive" result in 46 cases and "false positive" in two. The test was found to be "true negative" in 33 cases and "false negative" in 16 (table 1) Thus, the sensitivity of the test was $74 \%$ while the specificity was $94 \%$. The predictive value of a positive test was $96 \%$ and the predictive value of a negative test was $67 \%$.

All patients with AVNRT had an AH jump on EPS. There was no AH jump on EPS before or after ablation in any patient of AVRT, including the two who had a PR jump with the adenosine test.

Second degree or greater AV block was seen in 25 patients with AVNRT; this comprised 22/46 patients with a true positive test and 3/16 patients with a false negative test $(p<0.05)$. Thus, in patients with AVNRT, the absence of AV block was significantly associated with more false negative tests. This may be related to an inadequate dose of adenosine to block the fast AV node pathway. In patients with AVRT, five had a second degree or greater AV block, which comprised 4/33 true negative and $1 / 16$ false negative results.

AV nodal echo (retrograde $\mathrm{P}$ wave) was seen in three patients, all of whom had a true positive adenosine test. Latent pre-excitation was unmasked by adenosine in four patients with AVRT (fig 3). Adenosine injection initiated tachycardia in five patients, three with AVNRT (fig 4) and two with AVRT (fig $5)$.

Reverse PR jump - that is, a sudden shortening of PR interval during recovery from adenosine effect-was seen in 10 patients (fig 6). Of these, two had no PR jump. All 10 patients with reverse PR jump were found to have AVNRT by EPS.

Adenosine induced bradycardia was well tolerated by all patients. Thirty patients developed transient second or third degree AV block for a maximum duration of 2 minutes. Six 


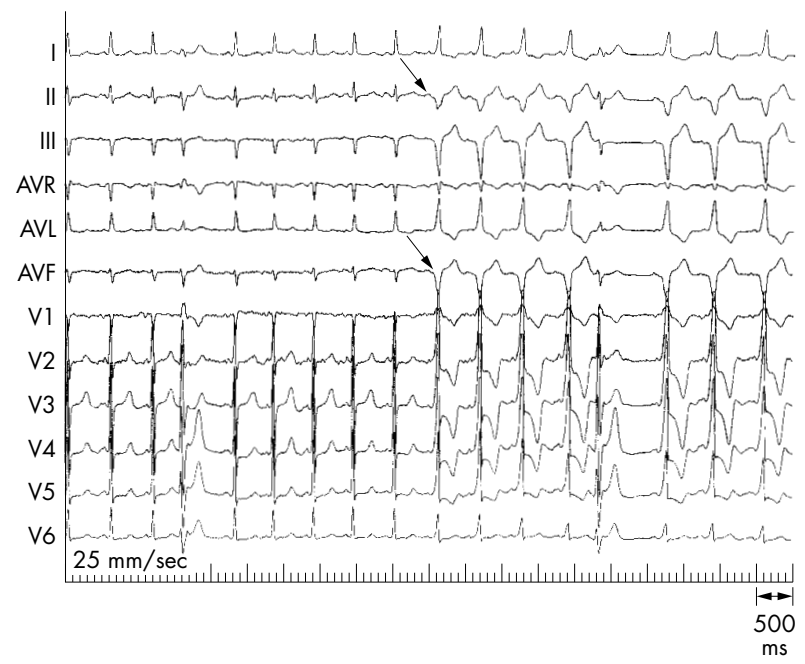

Figure 3 Unmasking of "latent pre-excitation" after adenosine injection, indicated by an arrow.

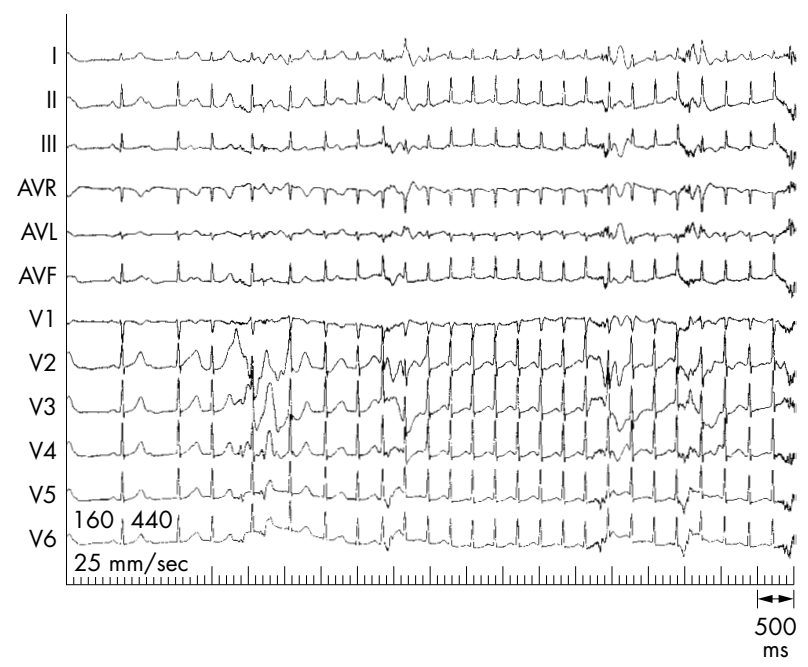

Figure 4 Initiation of atrioventricular nodal re-entrant tachycardia by adenosine, shown by an arrow, which is preceded by a PR jump (denoted by numbers in milliseconds).

patients had a long pause ( $>3$ seconds), the longest being of 7 seconds. However, none had syncope or required pacing or atropine.

All patients had minor side effects such as flushing, feeling of warmth, and giddiness but none developed bronchospasm or angina.

\section{DISCUSSION}

Among narrow QRS tachycardias, sinus node re-entrant tachycardia and atrial tachycardia can be differentiated with relative ease on the basis of surface 12 lead ECG. However, AVNRT and AVRT caused by concealed accessory pathways are difficult to differentiate from each other based only on surface ECG recorded at rest and during tachycardia. A precise non-invasive diagnosis would help in counselling the patient and preparing him or her for an EPS and radiofrequency ablation (which is increasingly becoming the treatment of choice in both cases) as regards the chances of success and complications such as AV block.

In the AV node with dual physiology, the antegrade fast pathway is more sensitive to the effects of adenosine than the slow pathway. A recent study of the effect of adenosine on antegrade fast and slow pathway conduction in patients with

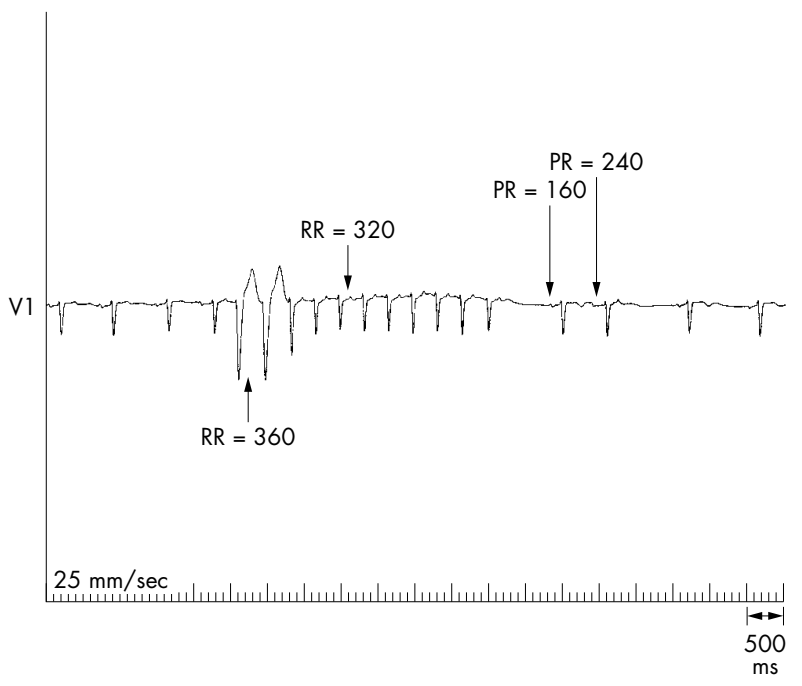

Figure 5 Atrioventricular re-entrant tachycardia induction during the adenosine test. A PR jump and an echo is seen after tachycardia. The left bundle morphology beats have longer RR interval than the narrow complex beats with a positive retrograde $\mathrm{P}$ wave in lead $\mathrm{V} 1$ suggestive of a left free wall concealed accessory pathway.

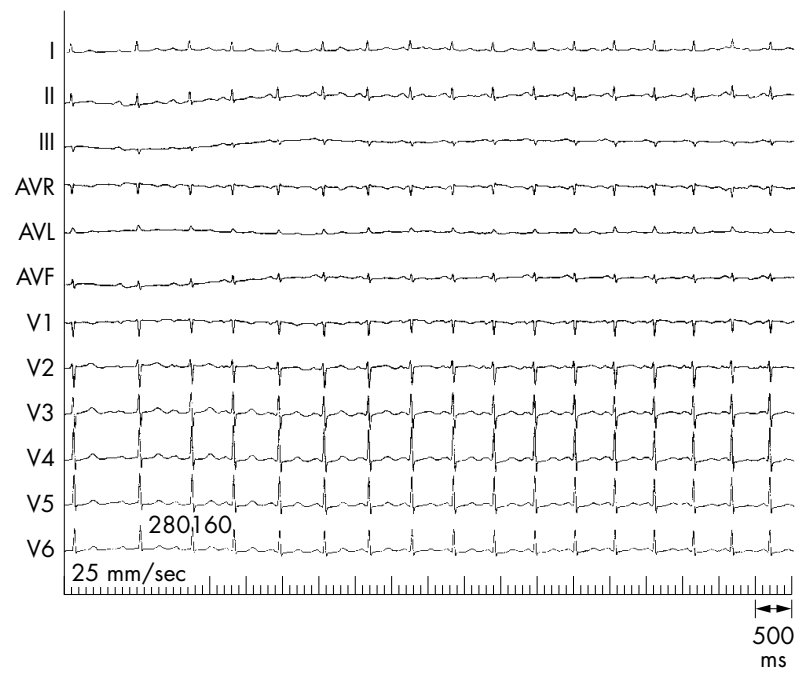

Figure 6 Twelve lead ECG showing a reverse PR jump following adenosine bolus during recovery. The numbers denote PR interval in milliseconds.

typical AVNRT found that a higher dose of adenosine was required to cause a block in the antegrade slow pathway than in the fast pathway. ${ }^{9}$ This difference in electrophysiological effects could prove to be useful in the non-invasive diagnosis of dual AV node physiology and could be used to differentiate between AVRT and AVNRT on surface ECG during sinus rhythm. In a patient with dual AV node physiology, when adenosine blocks only the fast pathway, AV conduction occurs over the slow pathway and is slower. This manifests itself as an increase of $\geqslant 50 \mathrm{~ms}$ in the AH interval on intracardiac recordings (AH jump) and in the PR interval on surface ECG (PR jump). An AV nodal echo is caused by retrograde conduction via the fast pathway and is therefore suggestive of AVNRT as a tachycardia mechanism.

Most patients (approximately 85\%) with typical AVNRT have demonstrable dual AV node physiology. ${ }^{10}$ In our study, dual AV node physiology was seen on EPS for all patients with AVNRT.

Dual AV nodal pathways occasionally can be noted as a concomitant but unrelated finding in patients with AVRT. ${ }^{11}$ 
Farshidi and colleagues ${ }^{12}$ in their study found an incidence of dual AV node pathways in patients with concealed bypass tract of nearly $40 \%$. However, none of our 35 AVRT patients had evidence of dual AV node physiology on EPS, not even the two who had a PR jump on the adenosine test.

The use of adenosine in terminating narrow QRS tachycardias and its role in the differential diagnosis of wide QRS tachycardias is well established. ${ }^{3}{ }^{4}$ Its role in the differential diagnosis of narrow QRS tachycardias has recently become the subject of interest. Belhassen and colleagues ${ }^{5}$ used ATP for showing dual AV nodal physiology during EPS in sinus rhythm. Tebbenjohanns and associates ${ }^{7}$ used incremental doses of adenosine, injected through the femoral vein during EPS, to differentiate AVNRT from other forms of supraventricular tachycardias. The mean (SD) dose required to show dual AV node physiology or to produce AV block was 10.3 (4.2) $\mathrm{mg}$. Hence, we chose to use $12 \mathrm{mg}$ of adenosine in a single bolus dose to make the test simpler, quicker, and more economical and yet have a good sensitivity and specificity. Intravenous administration through a peripheral vein at the bedside is likely to increase the clinical application of the test.

In our study, the test had a high positive predictive value of $96 \%$. Two AVRT patients out of 35, all without dual AV node physiology demonstrable on EPS, had a PR jump. There is no clear explanation for the finding of PR jump after adenosine test in patients without dual AV node physiology. The test had a negative predictive value of only $67 \%$. Thus, in 16 of 62 patients with AVNRT, adenosine failed to produce a PR jump or an AV nodal echo. This may be attributed to the use of a single dose of adenosine. Either the dose was too low to affect the fast as well as the slow pathway $(n=13)$ or it was so high that both the fast and the slow pathways were blocked simultaneously and produced an AV block $(\mathrm{n}=3)$.

An AV nodal echo was seen in three $(4.8 \%)$ patients with AVNRT and in none of the patients with AVRT. Belhassen and colleagues $^{5}$ reported a much higher incidence $(48 \%)$ of $\mathrm{AV}$ nodal echoes while Tebbenjohanns and associates ${ }^{7}$ found AV nodal echoes in only $23 \%$ of their patients. Our results may be due either to the difficulty in identifying retrograde $\mathrm{P}$ waves on surface ECG or to the use of a single dose of adenosine.

The reverse PR jump, which we found in 10 patients with AVNRT, has not been described earlier. We hypothesise that as the adenosine effect starts wearing off, the slow pathway recovers earlier than the fast pathway. When the fast pathway starts conducting, the PR interval shortens suddenly.

Four of the 35 patients with concealed accessory pathways developed manifest pre-excitation after adenosine injection. This is caused by the blocking of the AV node allowing conduction through the accessory pathway, thus unmasking the accessory pathway conduction. Garratt and colleagues ${ }^{13}$ described the use of intravenous adenosine to unmask latent pre-excitation during sinus rhythm.

Tachycardia was induced in three patients with AVNRT. The initiation of AVNRT by adenosine has been reported by Curtis and colleagues.' Adenosine may selectively block the antegrade fast pathway while allowing conduction in the slow pathway, thus setting up a substrate for re-entry.

Two patients with AVRT had induction of tachycardia with adenosine. This has been reported only once ${ }^{8}$ and its mechanism is unclear. Adenosine shortens atrial refractoriness and delays the conduction over the AV node. We hypothesise that as a consequence of these two effects, retrograde excitation of the atrium via the accessory pathway was made possible after adenosine injection, thus setting up a re-entry circuit.
Bradycardia and AV blocks following adenosine injection were short lasting and well tolerated by the patients. No patient required pacing or atropine. Minor side effectsnamely, flushing, a feeling of warmth, and giddinessoccurred in all patients but never lasted more than two minutes. No major adverse effects such as bronchospasm or angina were encountered in the study.

Despite the use of a single $12 \mathrm{mg}$ dose of adenosine, the sensitivity of the test in our study was $74 \%$, only slightly less than those obtained by Belhassen and colleagues ${ }^{5}(76 \%)$ and Tebbenjohanns and associates ${ }^{7}(76 \%)$, who had used incremental doses of ATP and adenosine, respectively. The specificity of our test was $94 \%$ compared with $100 \%$ and $95 \%$ reported by Belhassen and colleagues ${ }^{5}$ and Tebbenjohanns and associates, ${ }^{7}$ respectively.

\section{Conclusion}

A single dose (12 mg) of intravenous adenosine administered during sinus rhythm in patients with documented narrow QRS tachycardia without manifest pre-excitation can identify dual AV node physiology on surface ECG recording at the bedside. A positive adenosine test identified by a PR jump can differentiate AVNRT from AVRT with a high predictive value.

Authors' affiliations

S C Toal, B Uvajifdar, A K Gupta, A M Vora, Y Y Lokhandwala, Department of Cardiology, K E M Hospital, Mumbai, India

\section{REFERENCES}

1 Drury AN, Szent Gyorgyi A. The physiological activity of adenine compounds with especial reference to their action upon the mammalian heart. J Physiol 1929;68:213-37.

2 Somlo E. Adenosine triphosphate in paroxysmal tachycardia. Lancet 1955; i: 1125

3 Camm AJ, Garratt CJ. Adenosine and supraventricular tachycardia. N Engl J Med 1991;5:1621-9.

4 Sharma AD, Klein GJ, Yee R. Intravenous adenosine triphosphate during wide QRS complex tachycardia safety therapeutic efficacy and diagnostic utility. Am J Med 1990;88:337-43.

5 Belhassen B, Fish R, Glikson M, et al. Non invasive diagnosis of dual AV node physiology in patients with AV nodal reentrant tachycardia by administration of adenosine 5 triphophaste during sinus rhythm. Circulation 1998;98:47-53.

6 Zipes DP. The dual pathway concept in specific arrhythmias diagnosis and treatment. In: Braunwald E, ed. Heart disease: a text book of cardiovascular medicine, vol 1. Bangalore (Indian edition): W B Saunders, 1997:663

7 Tebbenjohanns J, Niehaus M, Korte T, et al. Non invasive diagnosis in patients with undocumented tachycardias: value of the adenosine test to predict AV nodal reentrant tachycardia. J Cardiovasc Electrophysiol 1999;10:916-23.

8 Belhassen B, Fish R, Viskin S, et al. Adenosine-5'-triphosphate test for the non-invasive diagnosis of concealed accessory pathway. J Am Coll Cardiol 2000:36:803-10.

9 Curtis AB, Belardinelli L, Woodard DA, et al. Induction of atrioventricular node reentrant tachycardia with adenosine: differential effects of adenosine on fast and slow atrioventricular node pathways. $J$ Am Coll Cardiol 1997;30:1778-84.

10 Josephson ME. Supraventricular tachycardias. In: Josephson ME, ed. Clinical cardiac electrophysiology: techniques and interpretations. Philadelphia: Lea \& Febiger, 1993:187.

11 Zipes DP. Specific arrhythmias: diagnosis and treatment. In: Braunwald E, ed. Heart disease: a textbook of cardiovascular medicine, 5th edn. Bangalore (Indian edition): W B Saunders, 1997:665.

12 Farshidi A, Josephson ME, Horowitz LN. Electrophysiologic characteristics of concealed bypass tracts. Am J Cardiol 1978;41:1052.

13 Garratt CJ, Antoniou A, Griffith M, et al. Use of intravenous adenosine in sinus rhythm as a diagnostic test for latent preexcitation. Am J Cardiol 1990;65:868-73. 